\title{
Silicon Carbide: A Gas Sensing Material for Selective Detection of $\mathrm{SO}_{2}{ }^{+}$
}

\author{
Andrea Gaiardo ${ }^{1,2, *}$, Barbara Fabbri ${ }^{1}$, Matteo Valt ${ }^{1}$, Vincenzo Guidi i , Cesare Malagù ${ }^{1}$, \\ Giulia Zonta ${ }^{1}$, Nicolò Landini ${ }^{1}$, Alessio Giberti ${ }^{3}$, Sandro Gherardi ${ }^{1}$ and Pierluigi Bellutti ${ }^{2}$ \\ 1 Department of Physics and Earth Sciences, University of Ferrara, 44122 Ferrara, Italy; \\ fbbbbr@unife.it (B.F.); matteo.valt@unife.it (M.V.); guidi@fe.infn.it (V.G.); malagu@fe.infn.it (C.M.), \\ zntgli@unife.it (G.Z.); lndncl@unife.it (N.L); ghrsdr@unife.it (S.G.) \\ 2 Micro Nano Facility, Bruno Kessler Foundation, 38123 Trento, Italy; bellutti@fbk.eu \\ 3 MIST E-R s.c.r.l., Via P. Gobetti 101, 40129 Bologna, Italy; giberti@fe.infn.it \\ * Correspondence: grdndr@unife.it \\ + Presented at the 5th International Symposium on Sensor Science (I3S 2017), Barcelona, Spain, \\ 27-29 September 2017.
}

Published: 29 November 2017

Silicon carbide $(\mathrm{SiC})$ is a long-time known material with exceptional mechanical properties. Ceramics obtained by sintering $\mathrm{SiC}$ grains are very hard and find application in car brakes, bulletproof vests and, in general, in high endurance applications. Moreover, its thermal strength and chemical stability are also extraordinary, maintaining stability at even above $1000{ }^{\circ} \mathrm{C}$, making it possible for use in high temperature applications. Considering these characteristics of silicon carbide, through the combination of stability and surface reactivity, it is possible to obtain a gas sensor suitable for commercial use. Therefore, this study was focused on the investigation of the chemoresistivity properties of $\mathrm{SiC}$ thick films in thermo-activation mode. Commercial Silicon Carbide nanopowder was characterized from the morphological, structural and chemical point of view. Then, it was screen-printed onto alumina substrates to obtain thick films and tested as chemiresistive gas sensors. The SiC layers were exposed to 13 gases belonging to different chemical classes, in concentrations within the ppm range and chosen through the Threshold Limit value. The sensors proved to be insensitive to almost all gases analysed, while they showed a significant response to sulphur dioxide in dry conditions. This cross selectivity increased in wet conditions, highlighting a possible sensing application of this material.

(C) 2017 by the authors. Licensee MDPI, Basel, Switzerland. This article is an open access article distributed under the terms and conditions of the Creative Commons Attribution (CC BY) license (http://creativecommons.org/licenses/by/4.0/) 